\title{
Rehabituación vestibular en la silla de Barany para el manejo de la aerocinetosis
}

| Fecha de recibido: 25 de noviembre del 2020 | Fecha de aprobación: 21 de abril del 2021 |

\author{
María Alejandra \\ Correa Guarín \\ Otorrinolaringóloga \\ Dirección de Medicina Aeroespacial \\ Fuerza Aérea Colombiana \\ Colombia \\ Rol de investigador: teórico \\ https://orcid.org/0000-0001-6015-144X \\ $\triangle$ correa10aleja@yahoo.com
}

\author{
Sonia Lucía \\ Jaimes Gómez \\ Médica de Aviación \\ Dirección de Medicina Aeroespacial, \\ Fuerza Aérea Colombiana \\ Colombia \\ Rol de investigador: teórico \\ http://orcid.org/0000-0002-3239-2788 \\ $\bowtie$ soniluja@gmail.com
}

\section{Claudia Marcela Mozo Madariaga \\ Audióloga}

Dirección de Medicina Aeroespacial, Fuerza Aérea Colombiana

Colombia

Rol de investigador: teórico https://orcid.org/0000-0001-7621-0555

$\bowtie$ claudimozom@yahoo.es

Cómo citar este artículo: Correa Guarín, M. A., Jaimes Gómez, S. L., \& Mozo Madariaga, C. M. (2021). Rehabituación vestibular en la silla de Barany para el manejo de la aerocinetosis. Ciencia y Poder Aéreo, 16(1), 149-157. https://doi.org/10.18667/cienciaypoderaereo.683 


\section{Rehabituación vestibular en la silla de Barany para el manejo de la aerocinetosis}

\section{Vestibular Adaptation in the Barany Chair for Handling Motion Sickness}

Resumen: La rehabituación es una parte de la terapia vestibular que sirve para mejorar el equilibrio, fundamental para el manejo de la aerocinetosis que se presenta en el personal de vuelo. Por esta razón, se decidió establecer un protocolo para realizar la rehabituación vestibular en la silla de Barany de la Dirección de Medicina Aeroespacial de la Fuerza Aérea Colombiana, con el fin de unificar criterios, según los parámetros de los protocolos definidos por otras fuerzas militares. Para esta demostración, se analizaron los casos de once pilotos que presentaron aerocinetosis, que fueron atendidos en la Dirección de Medicina Aeroespacial desde el 2016 hasta el 2020, y a quienes se les programaron sesiones para el tratamiento, de acuerdo con el protocolo. En estas sesiones se observó la recuperación de todos los pilotos, el mejoramiento de los síntomas y la recuperación de su actividad en vuelo. Adicionalmente se les explicaron las indicaciones que debían seguir para su reintegro laboral.

Palabras clave: enfermedad del movimiento; adaptación vestibular; silla de Barany.

Abstract: Adaptation makes part of vestibular therapy and serves to improve the functioning of the balance system, which is essential for managing motion sickness in flight personnel. For this reason, it was decided to establish a protocol for vestibular adaptation using the Barany chair property of the Colombian Air Force Directorate of Aerospace Medicine, in order to unify criteria around the parameters and protocols defined by other military forces. Eleven pilots affected by motion sickness and treated at the Directorate from 2016 to 2020 participated in the demonstration sessions, which were performed in accordance with the new protocol. During these sessions, researchers could observe the recovery of all pilots, who overcame the symptoms and could get back to their flight activity after receiving instructions on how to reincorporate to their duties.

Keyswords: Motion sickness; vestibular adaptation; Barany chair.

Resumo: A reabilitação é uma parte da terapia vestibular que serve para melhorar o funcionamento do sistema de equilíbrio, que é fundamental para o manejo da aerocinetose que se apresenta no pessoal de voo. Por esta razão, foi decidido estabelecer um protocolo para realizar a reabilitação vestibular na cadeira de Barany da Direção de Medicina Aeroespacial da Força Aérea Colombiana, com o fim de unificar critérios, segundo os parâmetros dos protocolos definidos por outras forças militares. Para esta demonstração, participaram onze pilotos que apresentaram aerocinetose e que se atenderam na Direção de Medicina Aeroespacial desde 2016 até 2020 , a quem foram realizadas sessões estabelecidas de acordo com o protocolo. Nestas sessões se observou a recuperação de todos os pilotos, com resolução dos sintomas e com recuperação de sua atividade em voo. Além disso, foram explicadas as indicações a seguir para a sua reintegração no mercado de trabalho.

Palavras-chave: doença do movimento; adaptação vestibular; cadeira de Barany. 
La enfermedad del movimiento es un conjunto de síntomas variados que ocurren en asociación con el movimiento de la persona o su entorno, en este caso, el vuelo. Algunos autores han mencionado que el estrés es un factor psicológico desencadenante de esta afección. Los síntomas que presentan son autonómicos e insidiosos: adormecimiento en las manos, bostezos frecuentes y disminución de la atención, los cuales progresan con la sensación de mareo, sudoración, palidez, salivación, cefalea, náuseas y vómito, lo que produce una incapacidad severa e incluso la pérdida del conocimiento. Estos síntomas generalmente desaparecen a las veinticuatro horas después del cese del estímulo.

La teoría más aceptada para la aerocinetosis es que se trata de un conflicto en el procesamiento de la información del sistema vestibular, visual y propioceptivo, dentro de un sistema sensorial multimodal cuya función está determinada por el movimiento relativo individual y de su ambiente, a la que se le ha llamado la hipótesis del mismatch neural o del desajuste neural, descrita por Benson (Correa \& Caputo 2014; Bertolini \& Straumann 2016; Lee et al, 2012). El grado de aerocinetosis se basa en los síntomas y en la incapacidad que genera al individuo el estímulo de movimiento en el vuelo - tabla 1- (Correa \& Caputo, 2014).

Uno de los pilares para el tratamiento de la cinetosis es la habituación vestibular; esta se fundamenta en un fenómeno fisiológico de compensación del sistema vestibular, que se basa en la repetición del estímulo generador de los síntomas. A través del aprendizaje y el reconocimiento, se logra la adaptación del sistema vestibular al suprimir la respuesta al estímulo (Riveros et al, 2007).

Ahora bien, la plasticidad neuronal vestibular se define como una modificación en los tipos de la comunicación entre dos células o dos redes neuronales, la cual permite que se produzca una compensación del sistema central por diferentes mecanismos como la habituación, la adaptación y la sustitución (Riveros et $a l, 2007)$.

La habituación es una técnica de rehabilitación vestibular descrita inicialmente por Cawthorne y Cooksey, y luego por Norre y De Weerdt. El principio de esta técnica se basa en la realización de una serie de ejercicios o estímulos repetitivos, cuya finalidad es desencadenar el cuadro de sensación vertiginosa, hasta que a través de la repetición se consiga la supresión de la respuesta, adaptación y reconocimiento del estímulo desencadenante. Su fundamento radica en la existencia de un conflicto sensorial derivado de la falta de congruencia en la información que llega por la vía vestibular, visual y propioceptiva, que se manifiesta como un cuadro vertiginoso. Mediante la repetición frecuente de la circunstancia desencadenante, se logra la habituación a la sensación vertiginosa hasta que se produce una adaptación (Novoa, 2019).

Este fenómeno de habituación tiene su impacto en el sistema nervioso central que, basado en la repetición de señales erróneas, conforma una nueva "imagen central”, resultado de la estimulación vestibular; finalmente, no se mostrará una información discordante. Al realizar esta repetición aparece un fenómeno de sustitución por aprendizaje de la capacidad de

Tabla 1

Grados de aerocinetosis y entrenamiento

\begin{tabular}{l|l|l|l}
\multicolumn{1}{c|}{ Grado I } & \multicolumn{1}{c|}{ Grado II } & \multicolumn{1}{c|}{ Grado III } \\
\hline $\begin{array}{l}\text { Síntomas leves: náuseas, } \\
\text { dispepsia, mareo leve }\end{array}$ & $\begin{array}{l}\text { Síntomas moderados: además de lo } \\
\text { anterior, sudoración y sialorrea }\end{array}$ & $\begin{array}{l}\text { Síntomas severos: aumenta la } \\
\text { intensidad de los síntomas y palidez }\end{array}$ & $\begin{array}{l}\text { Síntomas incapacitantes: emesis, } \\
\text { desorientación, alteración } \\
\text { conciencia }\end{array}$ \\
\hline Apoyo psicológico & Apoyo psicológico & Apoyo psicológico psicológico \\
\hline Ejercicios de respiración y relajación & Ejercicios de respiración y relajación & Ejercicios de respiración y relajación & Ejercicios de respiración y relajación \\
\hline $\begin{array}{l}2 \text { sesiones en silla de Barany } \\
\text { (un día) }\end{array}$ & $\begin{array}{l}5 \text { sesiones en silla de Barany } \\
\text { (dos días y medio) }\end{array}$ & $\begin{array}{l}\text { 7 sesiones en silla de Barany } \\
\text { (tres días y medio) }\end{array}$ & $\begin{array}{l}10 \text { sesiones en silla de Barany } \\
\text { (cinco días) }\end{array}$ \\
\hline
\end{tabular}

Fuente: Correa \& Caputo (2014) 
cada sujeto, para establecer nuevamente la función (López et al, 2003).

Una de las formas de realizar la rehabituación vestibular es por medio de la silla de Barany, nombrada así por el fisiólogo húngaro Robert Barany, Premio Nobel de Fisiología en 1914. Este es un equipo que se utiliza para entrenamiento en fisiología de vuelo, con el propósito de demostrar algunas ilusiones y efectos de la desorientación espacial que provienen del sistema vestibular, así como para realizar entrenamiento en adaptación fisiológica y rehabituación en personas que presenten cinetosis (Gonzalez-Aguado, 2018).

El test rotatorio es la base del estímulo generado en la silla de Barany, que permite conocer el comportamiento del sistema vestibular, fundamental en la inclusión del movimiento ocular mediante la vía del Reflejo Vestíbulo-Ocular (vor, por sus siglas en inglés) (Pérez et al, 2009 \& Sanz et al, 2013). En otras palabras, este produce un estímulo del canal semicircular horizontal que desencadena el reflejo vestíbulo-ocular, al usar la rotación de la silla.

Las principales indicaciones de este test de rotación son: determinar la hipofunción vestibular bilateral, las patologías centrales, la rehabituación vestibular y el entrenamiento fisiológico de vuelo (Pérez et al, 2009; Sanz et al, 2013).

El VOR es uno de los tres reflejos vestibulares y es el responsable de mantener la fijación de los ojos durante la rotación de la cabeza. Este reflejo se basa en el test rotatorio en el que se genera una rotación del ojo con una amplitud igual y opuesta a la dirección del movimiento de la cabeza, como resultado de la estimulación vestibular (Herdman \& Clendaniel, 2014). El reflejo tiene un componente angular que está vinculado por los canales semicirculares, encargados de compensar la rotación y estabilizar la mirada. Además, tiene un componente lineal, mediado por los otolitos, que compensan el movimiento de translación. Este reflejo de movimiento se utiliza al ver objetos cercanos y al mover la cabeza a alta frecuencia (Sánchez et al, 2014).

Las crestas ampulares de cada canal semicircular horizontal se disponen perpendicularmente al eje del canal. Estas están constituidas por células neuroepiteliales pilosas que presentan estereocilios, y uno de mayor tamaño llamado kinocilio, orientado hacia el utrículo en el canal horizontal, y hacia el canal en los canales verticales. Según la segunda ley de Ewald, las corrientes hacia la ampolla (ampulipetas) generan una descarga excitatoria y las ampulifugas una descarga inhibitoria, mientras que en los canales verticales ocurre lo contrario, pues las ampulipetas inhiben y las ampulifugas excitan (Pérez et al, 2009; Sanz et al, 2013).

Al mover la cabeza a la derecha, el vor genera que el flujo endolinfático desplace la cúpula a la izquierda, lo que produce un aumento de la descarga de las células ciliadas del canal horizontal derecho y decrece las descargas de las células ciliadas del canal horizontal izquierdo. El nervio vestibular trasmite la información a los núcleos vestibulares medial y superior y al cerebelo; el estímulo excitatorio es trasmitido hasta el núcleo oculomotor que estimula el músculo ocular recto medial ipsilateral derecho y el recto lateral contralateral izquierdo; posteriormente, el impulso inhibitorio se trasmite al núcleo del músculo recto lateral ipsilateral derecho y recto medial contralateral izquierdo y, de este modo, provoca el movimiento compensatorio a la izquierda (Herdman \& Clendaniel, 2014).

La respuesta vestibular a un estímulo impulsivo es un nistagmo, cuya velocidad en la fase lenta decrece en el tiempo, si se mantiene una velocidad constante del estímulo (Pérez et al, 2009; Sanz et al, 2013). Dicha respuesta tiene tres componentes: el primero es mecánico y depende del desplazamiento de la cúpula en contraposición a tres fuerzas del complejo cúpula-endolinfa (inercia, elasticidad y viscosidad), lo que hace que los canales semicirculares se vuelvan menos sensibles a los movimientos de oscilación. El segundo es de origen central y supone el procesamiento de la señal que llega del nervio, permitiendo que la señal inicial se prolongue en el tiempo y dure más, fenómeno que se denomina almacenamiento de velocidad, y que refleja la señal de entrada en un circuito de retroalimentación positiva. El tercer componente es la adaptación que tiene origen a nivel central, la cual permite que la respuesta decline progresivamente y no persevere de manera indefinida (Zuma E Maia et al., 2017). 


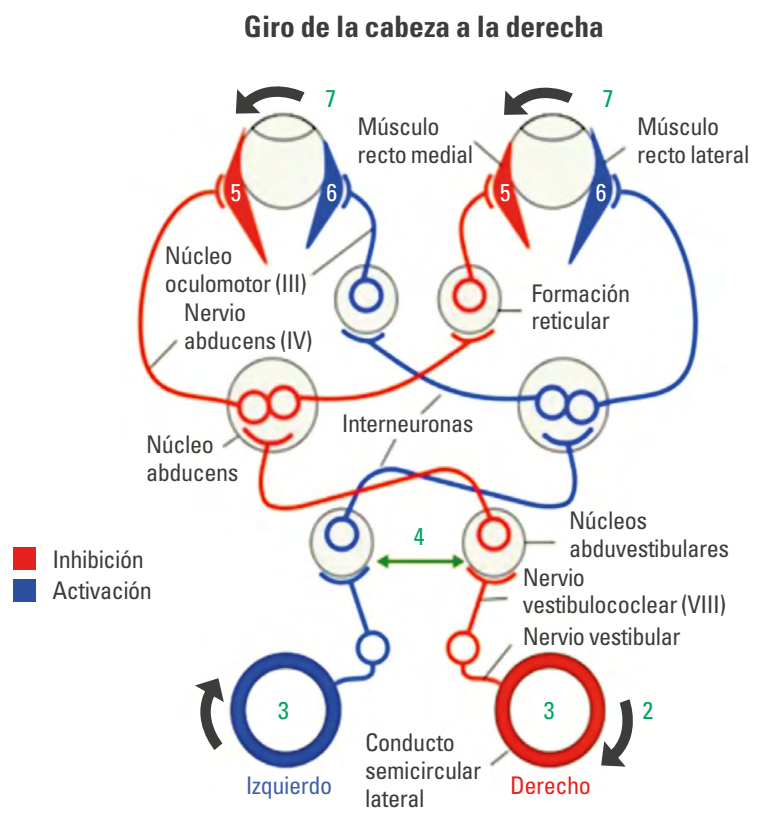

Figura 1. Reflejo Vestíbulo Ocular Fuente: Highstein (1998).

Asimismo, se pueden realizar dos pruebas con el test rotacional: una impulsiva, en donde se aplica una aceleración angular para alcanzar una velocidad constante durante un minuto, lo que provoca un nistagmo derecho si la rotación es horaria, y uno izquierdo si la rotación es antihoraria, y después de una desaceleración rápida se genera un nistagmo inverso (ilusión somatogiratoria). La otra prueba es la sinusoidal, en la que se emplean estímulos complejos variados en el eje vertical y horizontal, para lograr la estimulación adecuada. Al paciente se le flexiona la cabeza treinta grados para que los canales queden en el plano -ilusión de coriolisis- (Islam et al, 1997). Teniendo en cuenta estas premisas, se propuso aplicar un protocolo de rehabituación en la silla de Barany a los pilotos con aerocinetosis.

\section{Material y métodos}

Se realizó un estudio observacional, descriptivo y de corte transversal de los pacientes que asistieron a la Dirección de Medicina Aeroespacial desde el 2016 hasta el 2020. En total fueron once pacientes, hombres con aerocinetosis grado IV, con edades entre los 19 y 22 años, pilotos en formación, sin otras patologías asociadas, a quienes se les realizó el protocolo de rehabituación vestibular en la silla de Barany de Environmental Tectonics Corporation 1997, que es un simulador de ilusiones vestibulares. Se realizaron diez sesiones en total, dos diarias durante cinco días. Todos los pacientes regresaron a su actividad de vuelo al terminar la rehabituación y se encontró una mejoría en el cuadro que los hizo suspender su actividad de vuelo.

La silla de Barany está constituida por una cabina con una silla con pedales y un stick, un eje de montaje, y una consola de instrucción que tiene como objetivo dar un entrenamiento de fisiología y adaptación vestibular. Este equipo de ingeniería para entrenamiento fisiológico está conforme a los estándares militares utilizados para la preparación del personal de vuelo - figura 2-. A su vez, está diseñado para simular las ilusiones de vuelo, las cuales son replicadas por la rotación en el eje axial, y provocan una falsa sensación de movimiento y la ilusión de coriolisis. Esos efectos son inducidos a través de la rotación de la silla con las manecillas del reloj o en contra de ellas, cuya capacidad de rotación es ajustable de cero a veinticinco revoluciones por minuto, con un controlable rango de aceleración desde un nivel sublímite a setenta y cinco grados por segundo cuadrado, el cual simula el test de silla rotatoria.

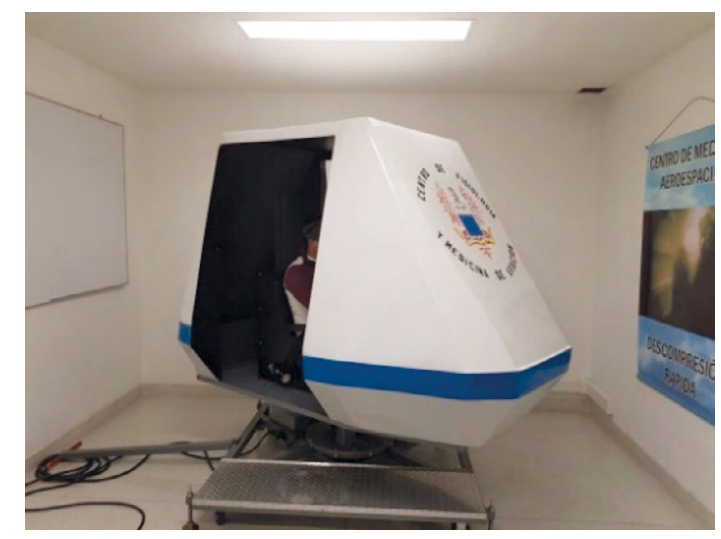

Figura 2. Silla de Barany, Environmental Tectonics Corporation 1997 Fuente: elaboración propia. 
Las ilusiones vestibulares que se pueden generar en la silla de Barany son las siguientes: ilusión somatogiratoria y cinco tipos diferentes de ilusión de coriolisis que se logran mediante movimientos de la cabeza en diferentes ejes, al tiempo que se lleva a cabo el movimiento de rotación de la silla, simulando el test de silla rotatoria.

Los síntomas que se presentan en los participantes durante el vuelo, con el estímulo en la silla, se gradúan a partir de la clasificación subjetiva de síntomas descrita por la Fuerza Aérea de los Estados Unidos (USAF por sus siglas en inglés), la cual se toma como referencia para realizar la graduación de los síntomas durante las sesiones de rehabituación vestibular, con el fin de determinar si pueden o no continuar con la terapia en la silla de Barany - ver tabla $2-$.

Tabla 2

Grados de los síntomas presentados por los alumnos durante el vuelo

\begin{tabular}{c|l}
$\begin{array}{c}\text { Grado } \\
\text { de mareo }\end{array}$ & \multicolumn{1}{c}{ Descripción } \\
\hline $1-3$ & $\begin{array}{l}\text { Refiere mínima sensación de náuseas } \\
\text { o malestar, no afecta sus actividades en el vuelo. }\end{array}$ \\
\hline $4-6$ & $\begin{array}{l}\text { Refiere moderada sensación de náuseas, } \\
\text { puede desviarse del plan de vuelo. } \\
\text { Ejemplo en la dirección y altura. }\end{array}$ \\
\hline $7-9$ & $\begin{array}{l}\text { Refiere mucha sensación de náuseas, detienen su } \\
\text { actividad de vuelo. Transfiere el control del vuelo. }\end{array}$ \\
\hline 10 & $\begin{array}{l}\text { Puede presentar vómito, lipotimia, pérdida } \\
\text { control del vuelo. }\end{array}$ \\
\hline
\end{tabular}

Fuente: Air Force (2020).

Para iniciar el entrenamiento de rehabituación vestibular, primero se plasmó la historia clínica de cada paciente, y se obtuvo la información sobre los síntomas, evolución, eventos relacionados con estos, síntomas asociados, eventos que generan la sensación y tratamientos recibidos. Además, se realizó la valoración por Otorrinolaringología, Medicina Aeroespacial y Psicología, y se efectuaron los estudios audiológicos y paraclínicos necesarios. Estas acciones permitieron determinar el grado de aerocinetosis que presentaba cada piloto y establecer el número de sesiones que se le realizarían a cada paciente (Correa \& Caputo, 2014).
Al iniciar las sesiones se les explicó a los pilotos qué es la aerocinetosis y se compartió información sobre la terapia vestibular, así como la forma como deberían respirar durante el desarrollo de esta. En cada sesión se hicieron progresivamente los ejercicios de ilusión somatogiratoria y coriolisis, aumentando su intensidad según la tolerancia del paciente; esta última fue valorada a través de los síntomas que presentaban durante el estímulo y clasificada en la tabla 2, si presentaban síntomas grado 7-9, se suspendía el ejercicio.

El objetivo es que el paciente soporte la terapia vestibular por un periodo de veinte minutos continuos, con síntomas que pueda controlar. Dicha terapia siempre debe ir acompañada con ejercicios de respiración, ya que la relajación producida por una respiración adecuada disminuye el estrés y los síntomas durante el ejercicio, lo que mejora la concentración en la silla de Barany. La técnica de respiración más usada consiste en una inspiración nasal profunda regular, en un solo movimiento, se retiene el aire y después se expulsa por nariz o boca. Los datos fueron tomados de la historia clínica de los pacientes y de lo registrado en cada sesión de terapia vestibular. Se realizó la base de datos en Excel con las mediciones requeridas en este programa.

\section{Resultados}

En el periodo comprendido desde el 2016 hasta el 2020, en la Dirección de Medicina Aeroespacial se atendieron once pacientes pilotos, alumnos de la FAC con aerocinetosis grado Iv. Inicialmente se les hizo una valoración por Otorrinolaringología, Medicina Aeroespacial y Psicología, para descartar patología vestibular o sistémica y determinar su motivación hacia la actividad de vuelo. El promedio de edad de este grupo de pacientes fue de 19 a 22 años, hombres en su totalidad, y cuyos síntomas limitaban su actividad de vuelo. Todos refirieron que en la infancia se mareaban con el olor de la gasolina y con el movimiento de diferentes tipos de transporte. Se les realizaron exámenes audiológicos con resultados normales, y laboratorios como 
hemograma, perfil lipídico, glicemia y la prueba de Hormona Estimulante de Tiroides (TSH, por sus siglas en inglés), que se encontraban dentro de parámetros normales.

Se les inició el protocolo de habituación vestibular en la silla de Barany a los once pacientes, con diez sesiones de veinte minutos, dos sesiones al día, durante las cuales se les sometía a estímulos somatogiros y coriolisis según la tolerancia del paciente y en forma progresiva; además, se les midió los síntomas que presentaban en las sesiones, de acuerdo con la clasificación de la tabla 2. En la figura 3 se describen los síntomas detectados en las sesiones uno, tres y diez. Allí se puede observar que a medida que el piloto se estimula, los síntomas disminuyen y es posible controlarlos.

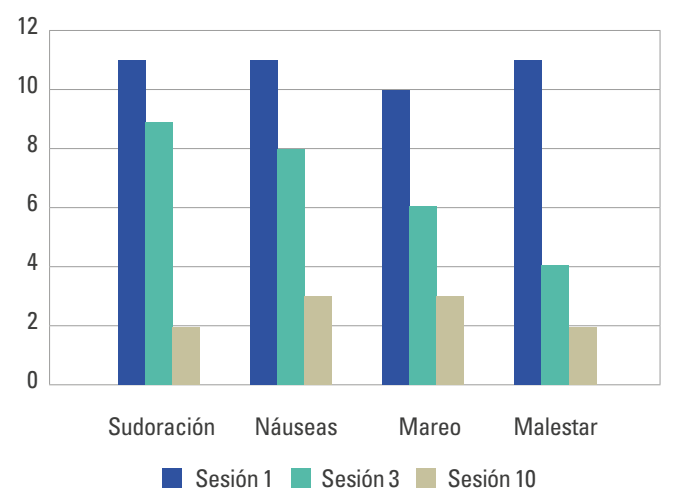

Figura 3. Síntomas presentados en las sesiones 1,3 y 10 de rehabituación Fuente: elaboración propia.

Además, se determinó que durante la terapia vestibular los síntomas descritos tuvieron una mayor intensidad con los estímulos de coriolisis, y que los pacientes reconocieron la importancia de conocer qué es la aerocinetosis y su fisiopatología, como estrategia para ayudarlos a controlar sus síntomas.

Al terminar la terapia de habituación vestibular, se les dio nuevamente la información del anexo 1 para continuar las actividades en su lugar de trabajo, y se les sugirió reiniciar sus actividades de vuelo en los siguientes tres días, después de terminar las sesiones. Después se hizo seguimiento a los once pacientes y se concluyó que todos retomaron sus actividades de vuelo sin ninguna limitación.

\section{Conclusión}

La aerocinetosis, o enfermedad del movimiento, es una alteración fisiológica presentada ante la percepción del movimiento, en este caso, generada por el vuelo. Esta enfermedad provoca discapacidad a los tripulantes en su actividad laboral. Además, se han identificado algunos factores predisponentes y de susceptibilidad individual. Uno de los pilares del tratamiento es la rehabituación vestibular, que se basa en la repetición del estímulo de movimiento, lo que ocasiona una supresión de la respuesta y, en consecuencia, un aprendizaje y acomodación al estímulo. Por lo tanto, se pudo observar que los pacientes mejoraron en el manejo de los síntomas y en el autocontrol de estos y después fueron reintegrados a sus labores de vuelo sin presentar ningún inconveniente.

De acuerdo con lo anterior, se evidencia el beneficio de la rehabituación vestibular en la aerocinetosis. Aunque en el ámbito académico todavía existe controversia sobre el origen de la fisiopatología de la aerocinetosis y su tratamiento, está claro que la habituación vestibular es efectiva para la resolución de los síntomas que se generan, y que la educación dada a los pilotos sobre esta condición, su prevención y manejo, es fundamental para obtener buenos resultados.

Declaración de conflicto de interés: Las autoras no manifiestan conflictos de interés institucionales ni personales.

\section{Referencias}

Air Force. (2020). Aerospace Physiological Training Program. Air Force Instruction AFI11-403.

López, O. A., Fernández, N. P. \& Sánchez, N. (2003). Rehabilitación vestibular. Revista de Medicina de La Universidad de Navarra, XLVII(4), 72-76. https://revistas.unav.edu/ index.php/revista-de-medicina/article/view/9064

González-Aguado, R. (2018). Prueba rotatoria. Técnica e interpretación. Revista ORL, 9(3), 215-219. https://doi. org/10.14201/orl.17717

Bertolini, G. \& Straumann, D. (2016). Moving in a Moving World: A Review on Vestibular Motion Sickness. Frontiers in 
Neurology, 7(14). https://www.frontiersin.org/articles/ 10.3389/fneur.2016.00014/full\#B2

Correa, M. A. \& Caputo, L. A. (2014). Aerocinetosis: más que enfermedad desadaptación. Ciencia y Poder Aéreo, 9(1), 1725. https://doi.org/10.18667/cienciaypoderaereo.129

Herdman, S. J. \& Clendaniel, R. (2014). Vestibular Rehabilitation. F.A. Davis Company. https://books.google.com. $\mathrm{co} /$ books?hl=es\&lr=\&id=tEEWBAAAQBAJ\&oi=fnd\&pg= PR3\&dq=Herdman

Highstein, S. M. (1998). Role of the flocculus of the cerebellum in motor learning of the vestibulo-ocular reflex. Otolaryngology--Head and Neck Surgery: Official Journal of American Academy of Otolaryngology-Head and Neck Surgery, 119(3), 212-220. https://doi.org/10.1016/ S0194-5998(98)70056-7

Koch, A., Cascorbi, I., Westhofen, M., Dafotakis, M., Klapa, S. \& Kuhtz-Buschbeck, J. P. (2018). The Neurophysiology and Treatment of Motion Sickness. Deutsches Artzblatt Online, 115(41). https://doi.org/10.3238/arztebl.2018.0687

Lee, Y. J., Shin, J. E., Park, M. S., Kim, J. M., Na, B. R., Kim, C.H. \& Park, H. J. (2012). Comprehensive Analysis of Head-Shaking Nystagmus in Patients with Vestibular Neuritis. Audiology and Neurotology, 17(4), 228-234. https://doi. org/10.1159/000336958

Lucertini, M., Verde, P. \& Trivelloni, P. (2013). Rehabilitation from Airsickness in Military Pilots: Long-Term Treatment Effectiveness. Aviation, Space, and Environmental Medicine, 84(11), 1.196-1.200. https://doi.org/10.3357/ asem.3509.2013

Novoa, C. I. (2019). Mecanismos neurofisiológicos de la rehabilitación vestibular. Revista de Otorrinolaringología y Cirugía de Cabeza y Cuello, 79(2), 240-247. https://doi. org/10.4067/s0718-48162019000200240

Pérez-Fernández, N., Boleas-Aguirre, M.S. \& Sanz-Martín, E. (2009). Prueba rotatoria. Atlas de pruebas vestibulares para especialistas en otorrinolaringología. Vanguard Gráfic.

Riveros, H., Correa G. C., Anabalón B, J. L. \& Aranís, J. C. (2007). Efectividad de la rehabilitación vestibular en una serie clínica. Revista de Otorrinolaringología y Cirugía de Cabeza y Cuello, 67(3), 229-236. https://doi.org/10.4067/ S0718-48162007000300004

Sánchez, C., Yañez R., Benito, J. I., Gordon C. R. \& Batuecas, A. (2014). Cinetosis. Revista de la Sociedad Otorrinolaringológica de Castilla y León, 5(28), 233-251. https:// gredos.usal.es/bitstream/handle/10366/124985/revis taorl201428_cinetosis.pdf?sequence=1\&isAllowed=y

Sanz Martín, E., Sánchez, E., Zschaeck Luzardo, C., Rodríguez R.M., Ramos González, O. \& Portero de la Plaza, A. (2013). VIII Congreso de la Asociación Madrileña de Otorrinolaringología: interpretación de pruebas vestibulares. $\mathrm{Lu}$ minar SL. 25-40.

Zuma E Maia, F. C., Cal, R., D’Albora, R., Carmona, S. \& Schubert, M. C. (2017). Head-shaking tilt suppression: a clinical test to discern central from peripheral causes of vertigo. Journal of Neurology, 264(6), 1.264-1.270. https://doi. org/10.1007/s00415-017-8524-x

\section{Anexo 1 \\ Aerocinetosis}

\section{Recomendaciones Dirección de Medicina Aeroespacial \\ María Alejandra Correa y Luz Amparo Caputo}

\section{¿Qué es la aerocinetosis?}

Es un conflicto sensorial que genera náuseas y mareo durante el movimiento y que depende del historial de mareos previos de los pacientes en el momento de utilizar medios de transporte o con olores fuertes como la gasolina. Esta condición también puede presentarse porepisodios deansiedad oasociarsea algunaenfermedad sistémica.

Según los síntomas que presentan los grados de aerocinetosis así:

\begin{tabular}{c|l|l} 
Grado & \multicolumn{1}{|c|}{ Síntomas } & \multicolumn{1}{|c}{ Limitación en actividades } \\
\hline 1 & $\begin{array}{l}\text { Náuseas, dolor estómago, } \\
\text { mareo leve. }\end{array}$ & $\begin{array}{l}\text { No limitación en sus } \\
\text { actividades de vuelo. }\end{array}$ \\
\hline 2 & $\begin{array}{l}\text { Náuseas, mareo, } \\
\text { sudoración, sialorrea. }\end{array}$ & $\begin{array}{l}\text { Realiza las actividades } \\
\text { de vuelo, pero evita los } \\
\text { movimientos fuertes. }\end{array}$ \\
\hline 3 & $\begin{array}{l}\text { Aumenta los síntomas } \\
\text { anteriores y surge el dolor en } \\
\text { la boca del estómago. }\end{array}$ & $\begin{array}{l}\text { Limita sus actividades } \\
\text { de vuelo. }\end{array}$ \\
\hline 4 & $\begin{array}{l}\text { Vómito, decaimiento, dolor } \\
\text { de cabeza, confusión, } \\
\text { desorientación. }\end{array}$ & $\begin{array}{l}\text { Limita actividades, requiere } \\
\text { atención médica. }\end{array}$ \\
\hline
\end{tabular}




\section{Hay factores que predisponen la aparición de estos síntomas}
1. Ansiedad, estrés.
2. Olores.
3. Ayuno, alimentación.
4. Temperatura.
5. Acrobacias en el vuelo.

\section{Tratamiento}

Se debe evitar el uso de medicamentos, por lo tanto, es necesario realizar los siguientes ejercicios en casa tres veces al día con diez repeticiones de cada uno.

Movimientos oculares:

1. Mirar de izquierda a derecha.

2. Mirar arriba y abajo.

3. Rotación ocular horaria y antihoraria.

Movimientos de la cabeza:

1. Flexión y extensión de la cabeza (mentón-esternón / dorso-nuca).

2. Rotación de la cabeza (izquierda-derecha-izquierda).

3. Inclinación lateral (oído-hombro).

4. Rotación completa horaria y antihoraria.

Movimientos de pie:

Primero con los ojos abiertos, posteriormente con los ojos cerrados.

1. Flexión del tronco con los pies juntos (mano derecha pie derecho, mano izquierda pie izquierdo).
2. Flexión del tronco con rotación de la cabeza (izquierda-derecha-izquierda).

3. Flexión del tronco con rotación del tronco con los pies separados (mano derecha-pie izquierdo/ mano izquierda- pie derecho).

4. Rotación del tronco horario y antihorario.

Movimientos de brazos y cuerpo:

1. Sentarse y girar la cintura a la derecha y luego izquierda.

2. Sentarse e inclinarse hacia adelante para recoger un objeto del piso, volver a la posición inicial, después agacharse y dejar el objeto en el suelo nuevamente.

\section{Indicaciones de alimentación}

1. Comer 203 horas antes de la actividad de vuelo.

2. Evitar alimentos ácidos.

3. Evitar bebidas carbonatadas.

4. Evitas bebidas oscuras (tinto, chocolate, té).

5. Evitar comidas grasosas.

6. Evitar el cigarrillo.

7. Mantener una hidratación adecuada.

8. Horas de descanso.

9. Tener una rutina de actividad física.

\section{Recomendaciones durante el vuelo}

1. Usar una buena técnica de respiración.

2. Evitar movimientos de cabeza fuertes.

3. Mover los dedos de los pies si los siente fríos o sudorosos.

4. Masticar chicle durante el vuelo.

5. Si presenta mareo, fijar la mirada en los instrumentos de la nave. 